\title{
Towards error bounds of the failure probability of elastic structures using reduced basis models
}

\author{
L. Gallimard ${ }^{1, *, \dagger}$, E. Florentin ${ }^{2}$ and D. Ryckelynck ${ }^{3}$ \\ ${ }^{1}$ Laboratoire Energétique Mécanique Electromagnétisme EA4416, Université Paris Nanterre, 50 rue de Sèvres, \\ Ville d'Avray, 92410, France \\ ${ }^{2}$ INSA-CVL/Universite d'Orléans - PRISME, 88, Boulevard Lahitolle, Bourges, F-18020, France \\ ${ }^{3}$ Centre des Matériaux, MINES ParisTech, PSL, Research University, CNRS UMR 7633, Paris, France
}

\begin{abstract}
SUMMARY
Structural reliability methods aim at computing the probability of failure of systems with respect to prescribed limit state functions. A common practice to evaluate these limit state functions is using Monte Carlo simulations. The main drawback of this approach is the computational cost, because it requires computing a large number of deterministic finite element solutions. Surrogate models, which are built from a limited number of runs of the original model, have been developed, as substitute of the original model, to reduce the computational cost. However, these surrogate models, while decreasing drastically the computational cost, may fail in computing an accurate failure probability. In this paper, we focus on the control of the error introduced by a reduced basis surrogate model on the computation of the failure probability obtained by a Monte Carlo simulation. We propose a technique to determine bounds of this failure probability, as well as a strategy of enrichment of the reduced basis, based on limiting the bounds of the error of the failure probability for a multi-material elastic structure.
\end{abstract}

KEY WORDS: $\quad$ structural reliability; finite element analysis; model reduction; reduced basis; error bounds; failure probability

\section{INTRODUCTION}

Many applications in structural analysis require taking into account stochastic properties of material, geometry, or loads. Given a probabilistic description of the stochastic properties of the structure (i.e., a random vector $\Theta$ associated with a probability density function $f_{\Theta}$ ), reliability analysis aims at computing the probability of failure of systems with respect to a prescribed limit state function $G$. The failure is defined as an event $F=\{G(\Theta) \leqslant 0\}$, and the failure probability $P_{f}$ is defined by $P_{f}=\operatorname{Prob}(\{G(\Theta) \leqslant 0\})=\int_{G(\theta) \leqslant 0} f_{\Theta}(\boldsymbol{\theta}) d \boldsymbol{\theta}$. The computation of this failure probability by a direct Monte Carlo simulation would necessitate between $10^{3}$ and $10^{6}$ finite element (FE) simulations (depending on the value of $P_{f}$ ) and is too computationally expensive in practice. In order to reduce the number of simulation runs, different alternatives have been proposed.

A first approach is the first-order reliability method and the second-order reliability method (SORM) $[1,2]$ that consists in building a simple analytical approximation of the limit-state function around the so-called design point followed by a direct estimation of the failure probability [3-9].

A second approach consists in building a surface response as a surrogate of the limit state function (quadratic response surfaces, polynomial chaos expansions, kriging surrogates, etc.) [10-14]. The Monte Carlo simulation can be then applied on this surrogate model.

*Correspondence to: L. Gallimard, LEME, Université Paris Nanterre, 50 rue de Sèvres, 92410 Ville d'Avray, France.

†E-mail: laurent.gallimard@u-paris10.fr 
A third approach consists in building a reduced-order model (ROM) of the complex FE model [15-19] and using the Monte Carlo method along with the ROM.

Three main sources of errors are introduced in a reliability analysis: an error due to the FE analysis, an error due to the approximations introduced by the method used to reduce the number of FE simulation runs, and finally an error due to the Monte Carlo process (or due to the analytical formula used to compute the failure probability for the first-order reliability method/second-order reliability method). Methods have been developed over many years to evaluate the quality of the FE analysis [20-22] and have been extended more recently to the stochastic framework [23-25]; the quality of the Monte Carlo simulation can be assessed via the central limit theorem. Error estimators have been developed for ROM applied to parametrized model [26-30] and for ROM applied to the estimation of mean values [17]. However, as far as we know, there is no work on the estimation of the error introduced by a reduced-order modeling on the computation of the failure probability $P_{f}$.

In this paper, we focus on the control of the error introduced by a reduced basis approximation [30] on the computation of the failure probability $P_{f}$. We propose to use the concept of error in the constitutive relation to provide bounds of the failure probability for a given reduced basis. In a first step, these bounds are obtained following the methodology proposed in [26] by extracting a reduced basis that is equilibrated in the FE sense from a classical reduced basis (which satisfies the kinematic boundary conditions). In a second step, we propose a strategy to construct this reduced basis along with the Monte Carlo simulation, in order to provide controlled bounds of the failure probability $P_{f}$.

The paper is organized as follows: Section 2 describes the problem to be solved. In Section 3, the reduced basis formulation used to solve the problem is presented. The technique used to compute bounds on quantities of interest is reminded in Section 4. Its extension to the bounding of a failure probability as well as the algorithm used to build the reduced basis are developed in Section 5 . Finally, Section 6 presents an application to multilayered structures in 2D plane elasticity.

\section{THE PROBLEM TO BE SOLVED}

\subsection{Linear elastic model}

Let us consider an elastic structure defined in a domain $\Omega$ bounded by $\Gamma$. The external actions on the structure are represented by a surface force density $\mathbf{T}$ defined over a subset $\Gamma_{N}$ of the boundary and a body force density $\mathbf{b}$ defined in $\Omega$. We assume that a prescribed displacement $\mathbf{u}=\mathbf{u}_{d}$ is imposed on $\Gamma_{D}=\partial \Omega-\Gamma_{N}$. The material is assumed to be linear elastic, being $\mathbf{C}$ the Hooke tensor. We consider that the problem is dependent of a vector $\theta \in \mathcal{D} \subset \mathbb{R}^{p}$ of uncertain system parameters. These parameters are characterized in a probabilistic manner by means of a joint probability density function $f(\boldsymbol{\theta})$. The problem can be formulated as follows: Find a displacement field $\mathbf{u} \in \mathcal{U}$ and a stress field $\sigma$ defined in $\Omega$ that verify

- the kinematic constraints

$$
\mathbf{u}(\mathbf{x}, \boldsymbol{\theta})=\mathbf{u}_{d}(\mathbf{x}) \text { on } \Gamma_{D}
$$

- the equilibrium equations

$$
\operatorname{div} \boldsymbol{\sigma}(\mathbf{x}, \boldsymbol{\theta})+\mathbf{b}(\mathbf{x}, \boldsymbol{\theta})=\mathbf{0} \text { in } \Omega \text { and } \boldsymbol{\sigma}(\mathbf{x}, \boldsymbol{\theta}) \mathbf{n}=\mathbf{T}(\mathbf{x}, \boldsymbol{\theta}) \text { in } \Gamma_{N},
$$

- the constitutive equation

$$
\sigma(\mathbf{x}, \theta)=\mathbf{C}(\mathbf{x}, \theta) \varepsilon(\mathbf{u}(\mathbf{x}, \theta)) \text { in } \Omega .
$$

$\mathbf{n}$ denotes the outer normal to $\Omega . \mathcal{U}$ is the space in which the displacement field is being sought; $\mathcal{U}^{0}$ is the space of the fields in $\mathcal{U}$ that are zero on $\Gamma_{D}$; and $\varepsilon(\mathbf{u})$ denotes the linearized deformation associated with the displacement: $[\varepsilon(\mathbf{u})]_{i j}=1 / 2\left(u_{i, j}+u_{j, i}\right)$. 
We assume that the Hooke tensor $\mathbf{C}$, the body forces $\mathbf{b}$, and the traction forces $\mathbf{T}$ can be decomposed as the sum of functions of $\boldsymbol{\theta}$ multiplied by deterministic functions of $\mathbf{x}$.

$$
\mathbf{C}(\mathbf{x}, \boldsymbol{\theta})=\sum_{q=1}^{Q^{c}} \Theta_{q}^{c}(\boldsymbol{\theta}) \overline{\mathbf{C}}_{q}(\mathbf{x}), \quad \mathbf{b}(\mathbf{x}, \boldsymbol{\theta})=\sum_{q=1}^{Q^{b}} \Theta_{q}^{b}(\boldsymbol{\theta}) \overline{\mathbf{b}}_{q}(\mathbf{x}) \quad \mathbf{T}(\mathbf{x}, \boldsymbol{\theta})=\sum_{q=1}^{Q^{T}} \Theta_{q}^{T}(\boldsymbol{\theta}) \overline{\mathbf{T}}_{q}(\mathbf{x}),
$$

where $\Theta_{q}^{c}, \Theta_{q}^{b}$, and $\Theta_{q}^{T}$ are known functions of $\boldsymbol{\theta}$; each $\overline{\mathbf{C}}_{q}(\mathbf{x})$ is a fourth-order tensor defined in $\Omega$; $\overline{\mathbf{b}}_{q}(\mathbf{x})$ are vector fields defined in $\Omega$; and $\overline{\mathbf{T}}_{q}(\mathbf{x})$ are vector fields defined on $\Gamma_{N}$.

\section{Remark}

This decomposition can be obtained by the use of the Karhunen-Loeve expansion [31] as proposed in [25].

\section{Remark}

For the sake of simplicity, assume that $\mathbf{u}_{d}$ is a deterministic value (i.e., $\mathbf{u}_{d}(x, \boldsymbol{\theta})=\mathbf{u}_{d}(x)$ ). For an interested reader, the development of a reduced basis algorithm where $\mathbf{u}_{d}$ is also described by a stochastic approach can be found in [27].

Furthermore, we assume that the inverse of the Hooke tensor can be decomposed as the sum of functions of $\boldsymbol{\theta}$ multiplied by deterministic functions of $\mathbf{x}$.

$$
\mathbf{C}^{-1}(\mathbf{x}, \boldsymbol{\theta})=\sum_{q=1}^{Q^{s}} \Theta_{q}^{s}(\boldsymbol{\theta}) \overline{\mathbf{S}}_{q}(\mathbf{x})
$$

where each $\overline{\mathbf{S}}_{q}(\mathbf{x})$ is a fourth-order tensor defined on $\Omega$.

The strong form of the problem (Equations (1)-(3)) is equivalent to the classical weak form formulation: Find $\mathbf{u} \in\left\{\mathbf{v} \in \mathcal{U} ; \mathbf{v}_{\mid \Gamma_{D}}=\mathbf{u}_{d}\right\}$ such that

$$
a\left(\mathbf{u}(\boldsymbol{\theta}), \mathbf{u}^{*} ; \boldsymbol{\theta}\right)=f\left(\mathbf{u}^{*} ; \boldsymbol{\theta}\right) \quad \forall \mathbf{u}^{*} \in \mathcal{U}^{0},
$$

where

$$
a\left(\mathbf{u}(\boldsymbol{\theta}), \mathbf{u}^{*} ; \boldsymbol{\theta}\right)=\int_{\Omega} \mathbf{C}(\boldsymbol{\theta}) \varepsilon(\mathbf{u}(\boldsymbol{\theta})): \varepsilon\left(\mathbf{u}^{*}\right) d \Omega \text { and } f\left(\mathbf{u}^{*} ; \boldsymbol{\theta}\right)=\int_{\Omega} \mathbf{b}(\boldsymbol{\theta}) \cdot \mathbf{u}^{*} d \Omega+\int_{\Gamma_{N}} \mathbf{T}(\boldsymbol{\theta}) \cdot \mathbf{u}^{*} d \Gamma .
$$

To compute the solution $\mathbf{u}(\boldsymbol{\theta})$ of Equation (6), an FE approximation $\mathbf{u}_{h}$ of $\mathbf{u}$ is introduced such that $\mathbf{u}_{h} \in \mathcal{U}_{h} \subset \mathcal{U}$. Let $\mathcal{P}_{h}$ be a partition of $\Omega$ into elements $E_{k}\left(k \in\left\{1, \ldots, N_{F E}\right\}\right)$. This partition formed by the union of all elements is assumed to coincide exactly with the domain $\Omega$, and any two elements are either disjoint or share a common edge. We assume that $\mathbf{u}_{d}$ can be represented by a displacement field in $\mathcal{U}_{h}$. The discretized problem is as follows: Find a displacement field $\mathbf{u}_{h}(\boldsymbol{\theta}) \in \mathcal{U}_{h}$ and a stress field $\sigma_{h}(\theta)$ defined in $\Omega$ that verify

- the kinematic constraints

$$
\mathbf{u}_{h}(\mathbf{x}, \boldsymbol{\theta})=\mathbf{u}_{d}(\mathbf{x}) \text { on } \Gamma_{D}
$$

- the FE equilibrium equations

$$
\int_{\Omega} \boldsymbol{\sigma}_{h}(\boldsymbol{\theta}): \varepsilon\left(\mathbf{u}_{h}^{*}\right) d \Omega=\int_{\Omega} \mathbf{b}(\boldsymbol{\theta}) \cdot \mathbf{u}_{h}^{*} d \Omega+\int_{\Gamma_{N}} \mathbf{T}(\boldsymbol{\theta}) \cdot \mathbf{u}_{h}^{*} d \Gamma \quad \forall \mathbf{u}_{h}^{*} \in \mathcal{U}_{h}^{0},
$$

- the constitutive equation

$$
\sigma_{h}(\mathbf{x}, \boldsymbol{\theta})=\mathbf{C}(\mathbf{x}, \boldsymbol{\theta}) \varepsilon\left(\mathbf{u}_{h}(\mathbf{x}, \boldsymbol{\theta})\right) \text { in } \Omega .
$$


The classical weak form formulation is find $\mathbf{u}_{h} \in\left\{\mathbf{v} \in \mathcal{U}_{h} ; \mathbf{v}_{\mid \Gamma_{D}}=\mathbf{u}_{d}\right\}$ such that

$$
a\left(\mathbf{u}_{h}(\boldsymbol{\theta}), \mathbf{u}_{h}^{*} ; \boldsymbol{\theta}\right)=f\left(\mathbf{u}_{h}^{*} ; \boldsymbol{\theta}\right) \quad \forall \mathbf{u}_{h}^{*} \in \mathcal{U}_{h}^{0},
$$

where $\mathcal{U}_{h}^{0}=\left\{\mathbf{v} \in \mathcal{U}_{h} ;\left.\mathbf{v}\right|_{\Gamma_{D}}=\mathbf{0}\right\}$.

\subsection{Quantity of interest}

The goal of the computation is often to obtain (or characterize) some specific quantity of interest (QoI). Thus, in this context, the QoI is also a random output. The QoI is denoted by $Q\left(\mathbf{u}_{h}(\boldsymbol{\theta}) ; \boldsymbol{\theta}\right)$, and it is given as a linear output of $\mathbf{u}_{h}(\boldsymbol{\theta})$, namely,

$$
Q\left(\mathbf{u}_{h}(\boldsymbol{\theta}) ; \boldsymbol{\theta}\right)=\int_{\Omega} \mathbf{b}_{\Sigma}(\boldsymbol{\theta}) \cdot \mathbf{u}_{h}(\boldsymbol{\theta}) d \boldsymbol{\Omega}+\int_{\Gamma_{N}} \mathbf{T}_{\Sigma}(\boldsymbol{\theta}) \cdot \mathbf{u}_{h}(\boldsymbol{\theta}) d \Gamma,
$$

where $\mathbf{b}_{\Sigma}$ and $\mathbf{T}_{\Sigma}$ are the given extractors that define the output of interest and can be decomposed as the sum of functions of $\boldsymbol{\theta}$ multiplied by deterministic functions of $\mathbf{x}$.

$$
\mathbf{b}_{\Sigma}(\mathbf{x}, \boldsymbol{\theta})=\sum_{q=1}^{Q^{b_{\Sigma}}} \Theta_{q}^{b_{\Sigma}}(\boldsymbol{\theta}) \overline{\mathbf{b}}_{\Sigma_{q}}(\mathbf{x}), \quad \mathbf{T}_{\Sigma}(\mathbf{x}, \boldsymbol{\theta})=\sum_{q=1}^{Q^{T_{\Sigma}}} \Theta_{q}^{T_{\Sigma}}(\boldsymbol{\theta}) \overline{\mathbf{T}}_{\Sigma_{q}}(\mathbf{x}) .
$$

\subsection{Structural reliability model}

In this section, we describe the problem of reliability assessment $[1,13,32]$. Let us denote by $S(\boldsymbol{\theta})=Q\left(\mathbf{u}_{h}(\boldsymbol{\theta}) ; \boldsymbol{\theta}\right)$ the computational output of interest and $R(\boldsymbol{\theta})$ the threshold associated with this computational output.

Classically, the structure failure state is defined by a limit state function

$$
G(\theta)=R(\theta)-S(\theta)
$$

such that

- $G(\theta)<0$ is a failure state for the structure;

- $G(\theta)=0$ is the limit state;

- $G(\theta)>0$ is a safe state for the structure.

According to this definition, the system fails when $G$ is lower or equal to zero. The failure probability $P_{f}$ is then given by

$$
P_{f}=\int_{\mathcal{D}_{f}=\left\{\boldsymbol{\theta} \in \mathbb{R}^{n} ; G(\boldsymbol{\theta}) \leqslant 0\right\}} f_{\Theta}(\boldsymbol{\theta}) d \boldsymbol{\theta},
$$

where $n$ is the dimension of the vector $\boldsymbol{\theta}$ and $f_{\Theta}(\boldsymbol{\theta})$ is a density probability function. The evaluation of the integral defined by Equation (13) is not easy because it represents a very small quantity and because the integration domain is defined implicitly. Monte Carlo simulation is the main approach to solving the reliability problem. Recasting Equation (13) as

$$
P_{f}=\int_{\mathbb{R}^{n}} \mathbf{1}_{\mathcal{D}_{f}}(\boldsymbol{\theta}) f_{\Theta}(\boldsymbol{\theta}) d \boldsymbol{\theta}=E\left[\mathbf{1}_{\mathcal{D}_{f}}\right]
$$

where $\mathbf{1}_{\mathcal{D}_{f}}$ is the failure indicator function being equal to one if $G(\boldsymbol{\theta}) \leqslant 0$ and zero otherwise. The probability of failure is equal to the expectation of $\mathbf{1}_{\mathcal{D}_{f}}$. The Monte Carlo technique consists in generating a number $N_{M C}$ of realizations $\boldsymbol{\theta}^{k}$ of the random vector $\Theta$ using the actual probability density function, then computing the Monte Carlo estimator $\hat{P}_{f}$ :

$$
\hat{P}_{f}=\frac{1}{N_{M C}} \sum_{k=1}^{N_{M C}} \mathbf{1}_{\mathcal{D}_{f}}\left(\boldsymbol{\theta}^{k}\right) .
$$


According to the central limit theorem, this estimator is asymptotically unbiased and normally distributed with variance

$$
\operatorname{Var}\left[\hat{P}_{f}\right]=\frac{\hat{P}_{f}\left(1-\hat{P}_{f}\right)}{N_{M C}-1} .
$$

When the failure probability is small, the coefficient of variation of the estimator is

$$
\delta=\frac{\sqrt{\operatorname{Var}\left[\hat{P}_{f}\right]}}{\hat{P}_{f}} \approx \frac{1}{\sqrt{N_{M C}} \sqrt{\hat{P}_{f}}} .
$$

From Equation (16), it can be seen that obtaining a coefficient of variation $\delta \leqslant 10 \%$ for a probability of failure of $10^{-n}$ requires about $10^{n+2} \mathrm{FE}$ simulations.

\section{REDUCED BASIS FORMULATION}

The purpose of a reduced basis method is to provide a fast evaluation $\mathbf{u}_{r b}(\boldsymbol{\theta})$ of the value of the displacement field $\mathbf{u}_{h}(\boldsymbol{\theta})$ and hence a fast evaluation $Q\left(\mathbf{u}_{r b}(\boldsymbol{\theta}) ; \boldsymbol{\theta}\right)$ of any $\mathrm{QoI} Q\left(\mathbf{u}_{h}(\boldsymbol{\theta}) ; \boldsymbol{\theta}\right)$. In Section 3.1, we recall a classical displacement approach that consists in performing a Galerkin projection onto a reduced basis space that is assumed to represent accurately the solutions of the problem to be solved. In this paper, the construction of this reduced basis space, which will be detailed in Section 5, is aimed to control the accuracy of the computed failure probability. To control this accuracy, we define and compute an error estimator that is an upper bound of the error on the QoI $\left|Q\left(\mathbf{u}_{h}(\boldsymbol{\theta}) ; \boldsymbol{\theta}\right)-Q\left(\mathbf{u}_{r b}(\boldsymbol{\theta}) ; \boldsymbol{\theta}\right)\right|$. The computation of this upper bound, as shown in Section 4, necessitates the construction of a stress field that satisfies the FE equilibrium equations (Equation (8)). In order to obtain a fast evaluation of this stress field, we propose in Section 3.2 to build, from the displacement reduced basis, a stress reduced basis that satisfies the FE equilibrium equations.

\subsection{Displacement approach}

Let $\mathbf{u}_{d i r} \in \mathcal{U}_{h}$ be a displacement field such that $\mathbf{u}_{d i r \mid \Gamma_{D}}=\mathbf{u}_{d}$. Let us introduce a set of samples in the parameter space $\mathcal{S}^{N_{s}}=\left\{\boldsymbol{\theta}_{1}, \ldots, \boldsymbol{\theta}_{N_{s}}\right\}$, where $\boldsymbol{\theta}_{i} \in \mathcal{D}$, and for each $\boldsymbol{\theta}_{n}$ compute a FE solution $\mathbf{u}_{h}^{0}\left(\boldsymbol{\theta}_{n}\right)$ in $\mathcal{U}_{h}^{0}$ described by the corresponding vector of nodal values $\mathbf{q}^{n}$.

$$
a\left(\mathbf{u}_{h}^{0}\left(\boldsymbol{\theta}_{n}\right), \mathbf{u}_{h}^{*} ; \boldsymbol{\theta}_{n}\right)=f\left(\mathbf{u}_{h}^{*} ; \boldsymbol{\theta}_{n}\right)-a\left(\mathbf{u}_{d i r}, \mathbf{u}_{h}^{*} ; \boldsymbol{\theta}_{n}\right) \quad \forall \mathbf{u}_{h}^{*} \in \mathcal{U}_{h}^{0} .
$$

On this space, we perform a Gram-Schmidt orthonormalizing process for the inner product $a(\mathbf{u}, \mathbf{v} ; \overline{\boldsymbol{\theta}})$ $(\overline{\boldsymbol{\theta}}$ being a fixed value of the random vector that is selected a priori as shown in Section 5.2) to build a reduced basis $\phi^{i}$. The reduced basis space is then defined by

$$
\mathcal{U}_{r b}^{0}=\operatorname{span}\left\{\boldsymbol{\phi}^{1}, \ldots, \boldsymbol{\phi}^{N_{s}}\right\} \subset \mathcal{U}_{h}^{0} .
$$

The choice of the samples in the parameter space $\mathcal{S}^{N_{s}}$ and of the associated reduced basis $\mathcal{U}_{r b}^{0}$ depends on the sampling strategy (see [30] for more details). In this paper, we propose a reduced basis strategy that permits to control the quality of the reliability analysis. This reduced basis strategy will be introduced in Section 5. The reduced basis approximation consists in solving Equation (6) in $\mathcal{U}_{r b}^{0}+$ $\left\{\mathbf{u}_{d i r}\right\}$. A key point to justify the use of the reduced basis approximation is that $N_{s}$ is assumed to be much smaller than the number of degree of freedom of the FE model $N_{F E}$ (i.e., $N_{s} \ll N_{F E}$ ).

$$
a\left(\mathbf{u}_{r b}^{0}(\boldsymbol{\theta}), \mathbf{u}_{r b}^{*} ; \boldsymbol{\theta}\right)=f\left(\mathbf{u}_{r b}^{*} ; \boldsymbol{\theta}\right)-a\left(\mathbf{u}_{d i r}, \mathbf{u}_{r b}^{*} ; \boldsymbol{\theta}\right) \quad \forall \mathbf{u}_{r b}^{*} \in \mathcal{U}_{r b}^{0} .
$$


Remark

The computation of $\mathbf{u}_{d i r}$ is performed off-line. The simplest choice is, for a given $\overline{\boldsymbol{\theta}} \in \mathcal{D}$, to fi nd $\mathbf{u}_{\text {dir }} \in\left\{\mathbf{v} \in \mathcal{U}_{h} ; \mathbf{v}_{\mid \Gamma_{D}}=\mathbf{u}_{d}\right\}$ such that

$$
a\left(\mathbf{u}_{d i r}, \mathbf{u}_{h}^{*} ; \overline{\boldsymbol{\theta}}\right)=0 \quad \forall \mathbf{u}_{h}^{*} \in \mathcal{U}_{h}^{0} .
$$

The reduced basis solution writes

$$
\mathbf{u}_{r b}(\boldsymbol{\theta})=\mathbf{u}_{d i r}+\sum_{i=1}^{N_{s}} \alpha_{i} \boldsymbol{\phi}^{i}
$$

The corresponding stress is defined using the constitutive equation

$$
\boldsymbol{\sigma}_{r b}(\boldsymbol{\theta})=\mathbf{C}(\boldsymbol{\theta}) \boldsymbol{\varepsilon}\left(\mathbf{u}_{d i r}\right)+\sum_{i=1}^{N_{s}} \alpha_{i} \mathbf{C}(\boldsymbol{\theta}) \boldsymbol{\varepsilon}\left(\boldsymbol{\phi}^{i}\right) .
$$

By introducing Equation (21) in Equation (19), we build the algebraic system

$$
[\mathbf{K}(\theta)][\boldsymbol{\alpha}]=[\mathbf{F}(\boldsymbol{\theta})] .
$$

The elements of $[\mathbf{K}(\boldsymbol{\theta})]$ and of $[\mathbf{F}(\boldsymbol{\theta})]$ are defined by

$$
K_{i j}(\boldsymbol{\theta})=a\left(\boldsymbol{\phi}^{i}, \boldsymbol{\phi}^{j} ; \boldsymbol{\theta}\right) \text { and } F_{i}(\boldsymbol{\theta})=f\left(\boldsymbol{\phi}^{i} ; \boldsymbol{\theta}\right) .
$$

Thanks to the decomposition (Equation (4)) of $\mathbf{C}, \mathbf{b}$, and $\mathbf{T}, K_{i j}$ and $F_{i}$ can be written as a linear combination of the functions $\Theta_{q}^{c}(\boldsymbol{\theta}), \Theta_{q}^{b}(\boldsymbol{\theta})$, and $\Theta_{q}^{T}(\boldsymbol{\theta})$

$$
K_{i j}(\boldsymbol{\theta})=\sum_{q=1}^{Q^{c}} \bar{K}_{i j q} \Theta_{c}^{q}(\boldsymbol{\theta}) \text { and } F_{i}(\boldsymbol{\theta})=\sum_{q=1}^{Q^{b}} \bar{b}_{i q} \Theta_{q}^{b}(\boldsymbol{\theta})+\sum_{q=1}^{Q^{T}} \bar{T}_{i q} \Theta_{q}^{T}(\boldsymbol{\theta}),
$$

where

$$
\bar{K}_{i j q}=\int_{\Omega} \overline{\mathbf{C}}_{q} \varepsilon\left(\boldsymbol{\phi}^{i}\right): \varepsilon\left(\boldsymbol{\phi}^{j}\right) d \Omega, \quad \bar{b}_{i q}=\int_{\Omega} \overline{\mathbf{b}}_{q} \cdot \boldsymbol{\phi}^{i} d \Omega, \text { and } \bar{T}_{i q}=\int_{\Gamma_{N}} \overline{\mathbf{T}}_{q} \cdot \boldsymbol{\phi}^{i} d \Gamma
$$

\subsection{Stress approach}

The first step consists in building a stress $\sigma_{\text {neu }}(\theta)$ that verifies the FE equilibrium $\forall \boldsymbol{\theta}$. Let us introduce two sets of displacement fields $\left(\mathbf{u}_{q}^{b}, \mathbf{u}_{q^{\prime}}^{T}\right) \in \mathcal{U}_{h}^{0}$, for $\left(q, q^{\prime}\right) \in\left\{1, \ldots, Q^{b}\right\} \times\left\{1, \ldots, Q^{T}\right\}$, such that

$$
a\left(\mathbf{u}_{q}^{b}, \mathbf{u}_{h}^{*} ; \overline{\boldsymbol{\theta}}\right)=\int_{\Omega} \overline{\mathbf{b}}_{q} \cdot \mathbf{u}_{h}^{*} d \Omega \text { and } a\left(\mathbf{u}_{q^{\prime}}^{T}, \mathbf{u}_{h}^{*} ; \overline{\boldsymbol{\theta}}\right)=\int_{\Gamma_{N}} \overline{\mathbf{T}}_{q^{\prime}} \cdot \mathbf{u}_{h}^{*} d \Gamma \quad \forall \mathbf{u}_{h}^{*} \in \mathcal{U}_{h}^{0} .
$$

The stress field $\boldsymbol{\sigma}_{\text {neu }}(\boldsymbol{\theta})$ defined by

$$
\boldsymbol{\sigma}_{\text {neu }}(\boldsymbol{\theta})=\sum_{q=1}^{Q^{b}} \Theta_{q}^{b}(\boldsymbol{\theta}) \mathbf{C}(\overline{\boldsymbol{\theta}}) \boldsymbol{\varepsilon}\left(\mathbf{u}_{q}^{b}\right)+\sum_{q^{\prime}=1}^{Q^{T}} \Theta_{q^{\prime}}^{T}(\boldsymbol{\theta}) \mathbf{C}(\overline{\boldsymbol{\theta}}) \boldsymbol{\varepsilon}\left(\mathbf{u}_{q^{\prime}}^{T}\right)
$$

verifies the equilibrium equation in the $\mathrm{FE}$ sense (Equation (28))

$$
\int_{\Omega} \boldsymbol{\sigma}_{\text {nеи }}(\boldsymbol{\theta}): \boldsymbol{\varepsilon}\left(\mathbf{u}_{h}^{*}\right) d \Omega=f\left(\mathbf{u}_{h}^{*} ; \boldsymbol{\theta}\right) \quad \forall \mathbf{u}_{h}^{*} \in \mathcal{U}_{h}^{0} .
$$

Let us consider the set of stress fields computed from the snapshot solutions (Equation (17))

$$
\boldsymbol{\sigma}_{r b}^{i}=\mathbf{C}\left(\boldsymbol{\theta}_{i}\right) \boldsymbol{\varepsilon}\left(\mathbf{u}_{h}^{0}\left(\boldsymbol{\theta}_{i}\right)+\mathbf{u}_{d i r}\right) \text { for } i \in\left\{1, \ldots, N_{s}\right\}
$$

and the set of stress fields defined by

$$
\Delta \sigma_{r b}^{i}=\sigma_{r b}^{i}-\sigma_{\text {neu }}\left(\theta_{i}\right)
$$


From Equations (17) and (28), it follows that $\left\{\Delta \boldsymbol{\sigma}_{r b}^{i}\right.$, for $\left.i \in\left\{1, \ldots, N_{s}\right\}\right\}$ is a set of stress fields equilibrated to zero in the FE sense. An orthonormal basis $\left\{\zeta^{1}, \ldots, \zeta^{\underline{N}_{s}}\right\}$ is built from $\Delta \sigma_{r b}^{i}$ by a Gram-Schmidt process with respect to an internal product $\int_{\Omega} \sigma_{1}: \mathbf{C}^{-1}(\boldsymbol{\theta}) \boldsymbol{\sigma}_{2} d \Omega$.

For each parameter $\boldsymbol{\theta}$, an admissible stress field $\boldsymbol{\sigma}_{r b}^{e}$ is sought in the reduced basis of equilibrated stress fields in the FE sense.

$$
\boldsymbol{\sigma}_{r b}^{e}(\boldsymbol{\theta})=\boldsymbol{\sigma}_{\text {neu }}(\boldsymbol{\theta})+\sum_{i=1}^{N_{s}} \beta_{i} \boldsymbol{\zeta}^{i} .
$$

The coefficients $\beta_{i}$ are computed in order to minimize a distance between the stress field computed in the reduced basis $\boldsymbol{\sigma}_{r b}(\boldsymbol{\theta})=\mathbf{C}(\boldsymbol{\theta}) \boldsymbol{\varepsilon}\left(\mathbf{u}_{r b}(\boldsymbol{\theta})\right)$ and $\boldsymbol{\sigma}_{r b}^{e}(\boldsymbol{\theta})$

$$
J\left(\beta_{1}, \ldots, \beta_{N_{s}}\right)=\int_{\Omega}\left(\boldsymbol{\sigma}_{r b}(\boldsymbol{\theta})-\boldsymbol{\sigma}_{r b}^{e}(\boldsymbol{\theta})\right): \mathbf{C}^{-1}(\boldsymbol{\theta})\left(\boldsymbol{\sigma}_{r b}(\boldsymbol{\theta})-\boldsymbol{\sigma}_{r b}^{e}(\boldsymbol{\theta})\right) d \boldsymbol{\Omega} .
$$

The minimization of Equation (32) leads to the algebraic system

$$
[\mathbf{S}(\theta)][\boldsymbol{\beta}]=[\mathbf{G}(\boldsymbol{\theta})] .
$$

The elements of $[\mathbf{S}(\boldsymbol{\theta})]$ and of $[\mathbf{G}(\boldsymbol{\theta})]$ are defined by

$$
S_{i j}=\int_{\Omega} \mathbf{C}^{-1}(\boldsymbol{\theta}) \zeta^{j}: \zeta^{i} d \Omega
$$

and

$$
G_{i}=\int_{\Omega} \boldsymbol{\zeta}^{i}:\left(\varepsilon\left(\mathbf{u}_{r b}\right)-\mathbf{C}^{-1}(\boldsymbol{\theta}) \boldsymbol{\sigma}_{\text {nеи }}(\boldsymbol{\theta})\right) d \boldsymbol{\Omega} .
$$

Thanks to the decompositions (Equations (4) and (5)), $S_{i j}$ and $G_{i}$ can be written as a linear combination of the functions $\Theta_{q}^{s}(\boldsymbol{\theta}), \Theta_{q}^{b}(\boldsymbol{\theta})$, and $\Theta_{q}^{T}(\boldsymbol{\theta})$

$$
\begin{gathered}
S_{i j}=\sum_{q=1}^{Q^{s}} \bar{S}_{i j q} \Theta_{q}^{s}(\boldsymbol{\theta}) \\
G_{i}=\bar{c}_{i 0}+\sum_{j=1}^{N_{s}} \bar{c}_{i j} \alpha_{j}-\sum_{j=1}^{Q^{s}}\left(\sum_{q=1}^{Q^{b}} \bar{b}_{i j q} \Theta_{j}^{s}(\boldsymbol{\theta}) \Theta_{q}^{b}(\boldsymbol{\theta})+\sum_{q=1}^{Q^{T}} \bar{T}_{i j q} \Theta_{j}^{s}(\boldsymbol{\theta}) \Theta_{q}^{T}(\boldsymbol{\theta})\right),
\end{gathered}
$$

where

$$
\bar{S}_{i j q}=\int_{\Omega} \mathbf{S}^{q} \zeta^{i}: \zeta^{j} d \Omega, \quad \bar{c}_{i 0}=\int_{\Omega} \zeta^{i}: \varepsilon\left(\mathbf{u}_{d i r}\right) d \Omega, \quad \bar{c}_{i j}=\int_{\Omega} \zeta^{i}: \varepsilon\left(\boldsymbol{\phi}^{j}\right) d \Omega
$$

and

$$
\bar{b}_{i j q}=\int_{\Omega} \zeta^{i}: \overline{\mathbf{S}}_{j} \mathbf{C}(\overline{\boldsymbol{\theta}}) \boldsymbol{\varepsilon}\left(\mathbf{u}_{q}^{b}\right) d \Omega, \quad \bar{T}_{i j q}=\int_{\Gamma_{N}} \boldsymbol{\zeta}^{i}: \overline{\mathbf{S}}_{j} \mathbf{C}(\overline{\boldsymbol{\theta}}) \varepsilon\left(\mathbf{u}_{q}^{T}\right) d \Gamma
$$

\section{BOUNDS ON THE QUANTITY OF INTEREST}

The computation of error bounds on linear QoI has been developed by many authors [22, 33-35] for measuring the gap between an exact QoI and a QoI computed from an FE analysis. There is less work concerning the error bounds between a QoI computed from an FE analysis and a QoI computed by a reduced modeling approach [36-38]. For any realization of the random vector $\boldsymbol{\theta}$, the error introduced by the reduced approximation is given by

$$
\mathbf{e}_{r b}(\boldsymbol{\theta})=\mathbf{u}_{h}(\boldsymbol{\theta})-\mathbf{u}_{r b}(\boldsymbol{\theta})
$$


because of the linearity assumption, one has

$$
Q\left(\mathbf{e}_{r b}(\boldsymbol{\theta}) ; \boldsymbol{\theta}\right)=Q\left(\mathbf{u}_{h}(\boldsymbol{\theta}) ; \boldsymbol{\theta}\right)-Q\left(\mathbf{u}_{r b}(\boldsymbol{\theta}) ; \boldsymbol{\theta}\right) .
$$

Following [33], we consider the following auxiliary problem: Find $\mathbf{u}_{h}^{\text {aux }} \in \mathcal{U}_{h}^{0}$ such that

$$
a\left(\mathbf{u}_{h}^{*}, \mathbf{u}_{h}^{a u x}(\boldsymbol{\theta}) ; \boldsymbol{\theta}\right)=Q\left(\mathbf{u}_{h}^{*}(\boldsymbol{\theta}) ; \boldsymbol{\theta}\right) \quad \forall \mathbf{u}_{h}^{*} \in \mathcal{U}_{h}^{0} .
$$

As $\mathbf{e}_{r b}(\boldsymbol{\theta}) \in \mathcal{U}_{h}^{0}$, we obtain a first representation of the error on the QoI

$$
Q\left(\mathbf{e}_{r b}(\boldsymbol{\theta}) ; \boldsymbol{\theta}\right)=a\left(\mathbf{e}_{r b}(\boldsymbol{\theta}), \mathbf{u}_{h}^{a u x}(\boldsymbol{\theta}) ; \boldsymbol{\theta}\right) .
$$

Let us compute an approximation of $\mathbf{u}_{h}^{a u x}$ in the reduced basis $\mathcal{U}_{r b}^{0}$

$$
a\left(\mathbf{u}_{r b}^{*}, \mathbf{u}_{r b}^{a u x}(\boldsymbol{\theta}) ; \boldsymbol{\theta}\right)=Q\left(\mathbf{u}_{r b}^{*}(\boldsymbol{\theta}) ; \boldsymbol{\theta}\right) \quad \forall \mathbf{u}_{r b}^{*} \in \mathcal{U}_{r b}^{0} ;
$$

by using Equations (10) and (19) and remarking that $\mathbf{u}_{r b}^{a u x}(\boldsymbol{\theta}) \in \mathcal{U}_{r b}^{0} \subset \mathcal{U}_{h}^{0}$, we obtain

$$
a\left(\mathbf{e}_{r b}(\boldsymbol{\theta}), \mathbf{u}_{r b}^{a u x}(\boldsymbol{\theta}) ; \boldsymbol{\theta}\right)=0 .
$$

A second representation of the error on the QoI is obtained by subtracting Equation (41) from Equation (39):

$$
Q\left(\mathbf{e}_{r b}(\boldsymbol{\theta}) ; \boldsymbol{\theta}\right)=a\left(\mathbf{e}_{r b}(\boldsymbol{\theta}), \mathbf{e}_{r b}^{a u x}(\boldsymbol{\theta}) ; \boldsymbol{\theta}\right), \text { where } \mathbf{e}_{r b}^{a u x}(\boldsymbol{\theta})=\mathbf{u}_{h}^{a u x}(\boldsymbol{\theta})-\mathbf{u}_{r b}^{a u x}(\boldsymbol{\theta}) .
$$

Following [35], the error in the QoI can be written as

$$
Q\left(\mathbf{e}_{r b}(\boldsymbol{\theta}) ; \boldsymbol{\theta}\right)=\frac{1}{4}\left\|s \mathbf{e}_{r b}+s^{-1} \mathbf{e}_{r b}^{a u x}\right\|_{u, \boldsymbol{\theta}}^{2}-\frac{1}{4}\left\|\mathbf{e}_{r b}-s^{-1} \mathbf{e}_{r b}^{a u x}\right\|_{u, \boldsymbol{\theta}}^{2},
$$

where $s$ is a scaling factor and $\|\mathbf{u}\|_{u, \theta}=a(\mathbf{u}, \mathbf{u} ; \boldsymbol{\theta})$. Then, for any $s$, we have the following bounding properties

$$
-\frac{1}{4}\left\|s \mathbf{e}_{r b}-s^{-1} \mathbf{e}_{r b}^{a u x}\right\|_{u, \boldsymbol{\theta}}^{2} \leqslant Q\left(\mathbf{e}_{r b}(\boldsymbol{\theta}) ; \boldsymbol{\theta}\right) \leqslant \frac{1}{4}\left\|s \mathbf{e}_{r b}+s^{-1} \mathbf{e}_{r b}^{a u x}\right\|_{u, \boldsymbol{\theta}}^{2} .
$$

In order to determine the bounds for the error in the QoI, Equation (44) indicates that it is sufficient to bound the global energy norms of $s \mathbf{e}_{r b}-s^{-1} \mathbf{e}_{r b}^{a u x}$ and $s \mathbf{e}_{r b}+s^{-1} \mathbf{e}_{r b}^{a u x}$. In this paper, the methodology proposed in [26] that is based on the concept of constitutive relation error estimator [39] is used to build bounds of these errors.

It can be shown that (we refer the reader to $[22,26,40]$ for more details)

$$
\begin{aligned}
& \left\|s \mathbf{e}_{r b}+s^{-1} \mathbf{e}_{r b}^{a u x}\right\|_{u, \boldsymbol{\theta}}^{2} \leqslant\left\|\left(s \boldsymbol{\sigma}_{r b}^{e}+s^{-1} \boldsymbol{\sigma}_{r b}^{a u x, e}\right)-\left(s \boldsymbol{\sigma}_{r b}+s^{-1} \boldsymbol{\sigma}_{r b}^{a u x}\right)\right\|_{\sigma, \boldsymbol{\theta}}^{2}, \\
& \left\|s \mathbf{e}_{r b}-s^{-1} \mathbf{e}_{r b}^{a u x}\right\|_{u, \boldsymbol{\theta}}^{2} \leqslant\left\|\left(s \boldsymbol{\sigma}_{r b}^{e}-s^{-1} \boldsymbol{\sigma}_{r b}^{a u x, e}\right)-\left(s \boldsymbol{\sigma}_{r b}-s^{-1} \boldsymbol{\sigma}_{r b}^{a u x}\right)\right\|_{\sigma, \boldsymbol{\theta}}^{2},
\end{aligned}
$$

where $\boldsymbol{\sigma}_{r b}$ and $\boldsymbol{\sigma}_{r b}^{a u x}$ are the stresses computed from the reduced basis solutions $\mathbf{u}_{r b}$ and $\mathbf{u}_{r b}^{a u x}$ through the constitutive equation (Equation (22)), and $\|\sigma\|_{\sigma, \theta}$ is defined by

$$
\|\sigma\|_{\sigma, \theta}^{2}=<\sigma, \sigma>_{\sigma, \theta} \text { with }\left\langle\sigma_{1}, \sigma_{2}>_{\sigma, \theta}=\int_{\Omega} \sigma_{1}: \mathbf{C}^{-1}(\boldsymbol{\theta}) \sigma_{2} d \Omega .\right.
$$

$\boldsymbol{\sigma}_{r b}^{e}(\theta)$ and $\boldsymbol{\sigma}_{r b}^{a u x, e}(\theta)$ are stress fields equilibrated in the FE sense that are computed, for a given value of $\boldsymbol{\theta}$, as proposed in Section 3.2

$$
\int_{\Omega} \boldsymbol{\sigma}_{r b}^{e}(\boldsymbol{\theta}): \varepsilon\left(\mathbf{u}_{h}^{*}\right) d \boldsymbol{\Omega}=f\left(\mathbf{u}_{h}^{*} ; \boldsymbol{\theta}\right) \text { and } \int_{\Omega} \boldsymbol{\sigma}_{r b}^{a u x, e}(\boldsymbol{\theta}): \varepsilon\left(\mathbf{u}_{h}^{*}\right) d \Omega=Q\left(\mathbf{u}_{h}^{*} ; \boldsymbol{\theta}\right) .
$$

The value that minimizes the bounds is

$$
s^{o p t}=\sqrt{\frac{\left\|\sigma_{r b}^{a u x, e}-\sigma_{r b}^{a u x}\right\|_{\sigma, \theta}}{\left\|\sigma_{r b}^{e}-\sigma_{r b}\right\|_{\sigma, \theta}}} .
$$


Finally, introducing $s^{o p t}$ in Equation (45) provides us the following computable bounds

$$
\begin{aligned}
& \frac{1}{4}\left\|s \mathbf{e}_{r b}+s^{-1} \mathbf{e}_{r b}^{a u x}\right\|_{u, \boldsymbol{\theta}}^{2} \leqslant e_{r b}^{+}(\boldsymbol{\theta}), \\
& \frac{1}{4}\left\|s \mathbf{e}_{r b}-s^{-1} \mathbf{e}_{r b}^{a u x}\right\|_{u, \boldsymbol{\theta}}^{2} \leqslant e_{r b}^{-}(\boldsymbol{\theta}),
\end{aligned}
$$

where

$$
\begin{aligned}
& e_{r b}^{+}(\boldsymbol{\theta})=\frac{1}{2}\left(\left\|\boldsymbol{\sigma}_{r b}^{e}-\sigma_{r b}\right\|_{\sigma, \boldsymbol{\theta}}\left\|\boldsymbol{\sigma}_{r b}^{a u x, e}-\sigma_{r b}^{a u x}\right\|_{\sigma, \theta^{+}}+<\sigma_{r b}^{e}-\sigma_{r b}, \sigma_{r b}^{a u x, e}-\sigma_{r b}^{a u x}>_{\sigma, \theta}\right) \\
& e_{r b}^{-}(\boldsymbol{\theta})=\frac{1}{2}\left(\left\|\boldsymbol{\sigma}_{r b}^{e}-\sigma_{r b}\right\|_{\sigma, \boldsymbol{\theta}}\left\|\boldsymbol{\sigma}_{r b}^{a u x, e}-\sigma_{r b}^{a u x}\right\|_{\sigma, \theta^{-}}<\sigma_{r b}^{e}-\sigma_{r b}, \sigma_{r b}^{a u x, e}-\sigma_{r b}^{a u x}>_{\sigma, \boldsymbol{\theta}}\right) .
\end{aligned}
$$

From Equations (37) and (44), it follows that the $\operatorname{QoI} Q\left(\mathbf{u}_{h}(\boldsymbol{\theta}) ; \boldsymbol{\theta}\right)$ is bounded by

$$
Q\left(\mathbf{u}_{r b}(\boldsymbol{\theta}) ; \boldsymbol{\theta}\right)-e_{r b}^{-}(\boldsymbol{\theta}) \leqslant Q\left(\mathbf{u}_{h}(\boldsymbol{\theta}) ; \boldsymbol{\theta}\right) \leqslant Q\left(\mathbf{u}_{r b}(\boldsymbol{\theta}) ; \boldsymbol{\theta}\right)+e_{r b}^{+}(\boldsymbol{\theta}) .
$$

\section{Remark}

If $S$ does not depend linearly on uh, bounding of the quantity interest is much more difficult and there are few works addressing the subject. In the case where $Q$ is an $L_{2}$ norm of the stress or a mean von Mises stress over a subdomain, it is possible to derive approximate upper bounds as shown in [41].

\section{ERROR BOUNDS ON THE FAILURE PROBABILITY AND REDUCED BASIS ALGORITHM}

In the following section, let us assume for the sake of simplicity that the threshold $R$ is a constant and that the limit state function defined in Equation (12) is

$$
G(\boldsymbol{\theta})=R(\boldsymbol{\theta})-Q\left(\mathbf{u}_{h}(\boldsymbol{\theta}) ; \boldsymbol{\theta}\right) ;
$$

the failure domain $\mathcal{D}_{f}$ is defined by

$$
\mathcal{D}_{f}=\left\{\boldsymbol{\theta} ; R(\boldsymbol{\theta})-Q\left(\mathbf{u}_{h}(\boldsymbol{\theta}) ; \boldsymbol{\theta}\right) \leqslant 0\right\} .
$$

The failure probability computed by a Monte Carlo method is given by Equation (15)

$$
\hat{P}_{f}=\frac{1}{N_{M C}} \sum_{k=1}^{N_{M C}} \mathbf{1}_{\mathcal{D}_{f}}\left(\boldsymbol{\theta}^{k}\right) .
$$

\subsection{Error bounds on the failure probability}

The approximate limit state function computed from the reduced basis analysis is

$$
G_{r b}(\boldsymbol{\theta})=R(\boldsymbol{\theta})-Q\left(\mathbf{u}_{r b}(\boldsymbol{\theta}) ; \boldsymbol{\theta}\right) .
$$

The reduced basis failure domain is defined by

$$
\mathcal{D}_{f}^{r b}=\left\{\boldsymbol{\theta} ; R(\boldsymbol{\theta})-Q\left(\mathbf{u}_{r b}(\boldsymbol{\theta}) ; \boldsymbol{\theta}\right) \leqslant 0\right\} .
$$

The approximate failure probability computed by a Monte Carlo method is given by

$$
\hat{P}_{f}^{r b}=\frac{1}{N_{M C}} \sum_{k=1}^{N_{M C}} \mathbf{1}_{\mathcal{D}_{f}^{r b}}\left(\theta^{k}\right),
$$

and from Equation (48), the following computable bounds of the limit state function $G(\theta)$ are obtained

$$
G_{r b}^{-}(\boldsymbol{\theta}) \leqslant G(\boldsymbol{\theta}) \leqslant G_{r b}^{+}(\boldsymbol{\theta}),
$$


where

$$
G_{r b}^{-}(\boldsymbol{\theta})=G_{r b}(\boldsymbol{\theta})-e_{r b}^{+}(\boldsymbol{\theta}) \text { and } G_{r b}^{+}(\boldsymbol{\theta})=G_{r b}(\boldsymbol{\theta})+e_{r b}^{-}(\boldsymbol{\theta}) .
$$

Let us introduce the failure domain associated with the lower bound and the upper bound of the limit state function and the associated failure probabilities

$$
\begin{gathered}
\mathcal{D}_{f}^{+}=\left\{\boldsymbol{\theta} ; G_{r b}(\boldsymbol{\theta})-e_{r b}^{+}(\boldsymbol{\theta}) \leqslant 0\right\} \text { and } \mathcal{D}_{f}^{-}=\left\{\boldsymbol{\theta} ; G_{r b}(\boldsymbol{\theta})+e_{r b}^{-}(\boldsymbol{\theta}) \leqslant 0\right\}, \\
\hat{P}_{f}^{+}=\frac{1}{N_{M C}} \sum_{k=1}^{N_{M C}} \mathbf{1}_{\mathcal{D}_{f}^{+}}\left(\boldsymbol{\theta}^{k}\right) \text { and } \hat{P}_{f}^{-}=\frac{1}{N_{M C}} \sum_{k=1}^{N_{M C}} \mathbf{1}_{\mathcal{D}_{f}^{-}}\left(\boldsymbol{\theta}^{k}\right) .
\end{gathered}
$$

As $\mathcal{D}_{f}^{-} \subset \mathcal{D}_{f} \subset \mathcal{D}_{f}^{+}$, we obtain the following bounds for the failure probability

$$
\hat{P}_{f}^{-} \leqslant \hat{P}_{f} \leqslant \hat{P}_{f}^{+} \text {. }
$$

\section{Remark}

It must be noticed that the obtained bounds concern the failure probability $\hat{P}_{f}$ computed from a Monte Carlo simulation. The quality of the Monte Carlo simulation will be classically assessed in the examples presented in Section 6 via the central limit theorem and the computation of a confidence interval.

Furthermore, to control the reduced basis algorithm, an error estimator $\varepsilon_{G}(\theta)$, which is an upper bound on the error on the limit state function $e_{G}(\theta)=\left|G(\theta)-G_{r b}(\theta)\right|$, is introduced

$$
e_{G}(\boldsymbol{\theta}) \leqslant \varepsilon_{G}(\boldsymbol{\theta})=\max \left(e_{r b}^{-}(\boldsymbol{\theta}), e_{r b}^{+}(\boldsymbol{\theta})\right) .
$$

\section{Properties:}

1. If for all $\boldsymbol{\theta}^{k}, \varepsilon_{G}\left(\theta^{k}\right)=0$ then $\hat{P}_{f}^{-}=\hat{P}_{f}^{+}=\hat{P}_{f}$.

2. If $\hat{P}_{f}^{-}=\hat{P}_{f}^{+}$, then $\varepsilon_{G}\left(\theta^{k}\right)=0$ for all $\theta^{k}$ and $\hat{P}_{f}=\hat{P}_{f}^{-}=\hat{P}_{f}^{+}$.

\subsection{Reduced basis algorithm}

The initial step of the algorithm consists in computing a displacement field $\mathbf{u}_{d i r}$ satisfying the boundary conditions (Equation (20)) and a stress field $\boldsymbol{\sigma}_{\text {nеu }}(\boldsymbol{\theta})$ satisfying the equilibrium in the FE sense (Equations (26) and (27)). The computation of $\mathbf{u}_{d i r}$ and $\boldsymbol{\sigma}_{\text {neu }}(\boldsymbol{\theta})$ involves the choice of a particular value of $\boldsymbol{\theta}$ denoted $\overline{\boldsymbol{\theta}}$; a simple choice is to use the mean value of the random vector $\overline{\boldsymbol{\theta}}=E[\boldsymbol{\theta}]$. This value will also be used in the Gram-Schmidt orthonormalizing algorithm. The Monte Carlo simulation process starts generating a sample of $\boldsymbol{\theta}$ denoted $\boldsymbol{\theta}_{1}$, which is used to compute the initial vector of the displacement reduced basis $\phi^{1}=\mathbf{u}_{h}^{0}\left(\boldsymbol{\theta}_{1}\right)$ from Equation (17) and the initial vector of the stress reduced basis $\zeta^{1}=\Delta \sigma_{r b}^{1}$ from Equations (29)-(30).

From this initial reduced basis, the idea is to improve the reduced basis during the Monte Carlo simulation process, increasing the number of elements in the reduced basis only when it is required. An admissibility criterion is required to control the algorithm. A prescribed tolerance $\tau$ is set. The reduced basis will be improved for a given Monte Carlo sample $\boldsymbol{\theta}_{i}$ if the state of the structure (safe or failure) cannot be certified and the error estimator on the limit state function $\varepsilon_{G}\left(\boldsymbol{\theta}_{i}\right)$ is greater than $\tau$. The algorithm is described in Algorithm 1.

Let us denote by $\mathcal{U}_{r b}^{0, N}=\operatorname{span}\left\{\boldsymbol{\phi}^{1}, \ldots, \boldsymbol{\phi}^{N}\right\}$ and $\mathcal{S}_{r b}^{0, N}=\operatorname{span}\left\{\boldsymbol{\zeta}^{1}, \ldots, \zeta^{N}\right\}$ the reduced basis spaces that have been generated along with the $n$ Monte Carlo samples $\boldsymbol{\theta}_{i}(i \in\{1, \ldots, n\})$. For the next Monte Carlo sample $\boldsymbol{\theta}_{n+1}$, an approximate solution $\mathbf{u}_{r b}\left(\boldsymbol{\theta}_{n+1}\right)$ of $\mathbf{u}_{h}\left(\boldsymbol{\theta}_{n+1}\right)$ is computed in the reduced basis space $\mathcal{U}_{r b}^{0, N}$. The approximate limit state function $G_{r b}\left(\boldsymbol{\theta}_{n+1}\right)$ is computed as well as its upper bound $G_{r b}^{+}\left(\boldsymbol{\theta}_{n+1}\right)$ and lower bound $G_{r b}^{-}\left(\boldsymbol{\theta}_{n+1}\right)$ (Equation (52)). In order to estimate the failure probability, we need to count the number of samples that belongs to the failure domain. Let us denote $N_{f}^{r b}$ the number of samples $\boldsymbol{\theta}_{i}$ where $G_{r b}\left(\boldsymbol{\theta}_{i}\right) \leqslant 0, N_{f}^{+}$the number of samples where $G_{r b}^{-}\left(\boldsymbol{\theta}_{i}\right) \leqslant 0$, and $N_{f}^{-}$the number of samples where $G_{r b}^{+}\left(\boldsymbol{\theta}_{i}\right) \leqslant 0$. Three cases need to be distinguished: 


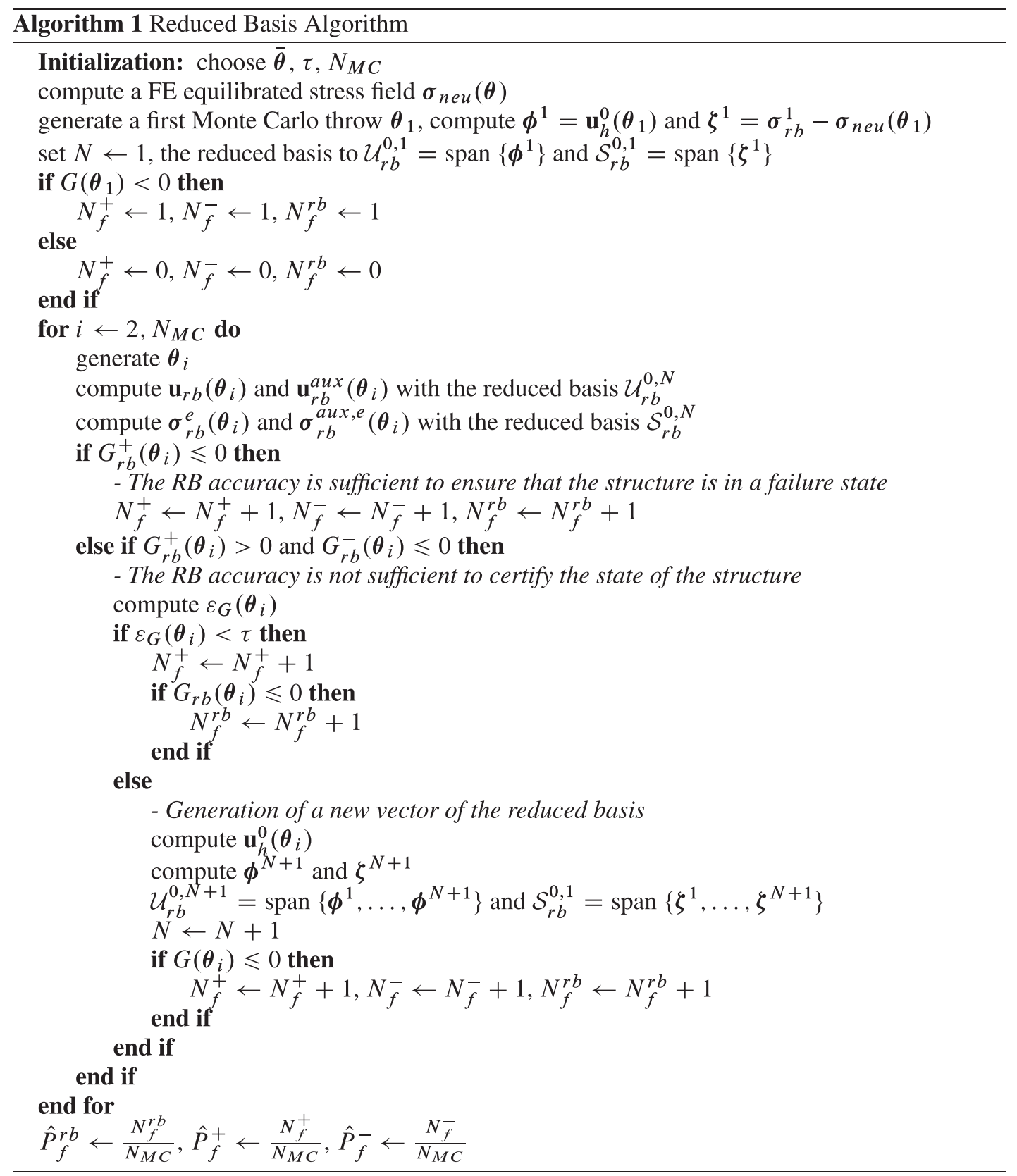

- If $G_{r b}^{-}\left(\boldsymbol{\theta}_{n+1}\right)>0$, then $G\left(\boldsymbol{\theta}_{n+1}\right)>0$ (Equation (52)), and the accuracy of the reduced basis approximation is sufficient to ensure that the structure is in a safe state;

- if $G_{r b}^{+}\left(\boldsymbol{\theta}_{n+1}\right) \leqslant 0$, then $G\left(\boldsymbol{\theta}_{n+1}\right) \leqslant 0$ (Equation (52)), and the accuracy of the reduced basis approximation is sufficient to ensure that the structure is in a failure state;

- if $G_{r b}^{-}\left(\boldsymbol{\theta}_{n+1}\right) \leqslant 0$ and $G_{r b}^{+}\left(\boldsymbol{\theta}_{n+1}\right)>0$, then the accuracy of the reduced basis approximation is not sufficient to certify the state of the structure.

In the first and second cases, there is no need to improve the reduced basis and the Monte Carlo simulations are continued by generating the next sample. In the third case, the reduced basis is improved only if the error estimator on the limit state function is greater than a fixed tolerance $\varepsilon_{G}\left(\boldsymbol{\theta}_{n+1}\right) \geqslant \tau$ : Equation (17) is solved in the FE space, and new reduced basis spaces are constructed $\mathcal{U}_{r b}^{0, N+1}=\operatorname{span}\left\{\boldsymbol{\phi}^{1}, \ldots, \boldsymbol{\phi}^{N+1}\right\}$ and $\mathcal{S}_{r b}^{0, N+1}=\operatorname{span}\left\{\boldsymbol{\zeta}^{1}, \ldots, \boldsymbol{\zeta}^{N+1}\right\}$. 
- If a new vector of the reduced basis is generated from $\boldsymbol{\theta}_{i}$, then $\mathbf{u}_{h}\left(\boldsymbol{\theta}_{i}\right)$ is computed and no error due to the reduced basis approximation is introduced: $G\left(\boldsymbol{\theta}_{i}\right)=G_{r b}^{+}\left(\boldsymbol{\theta}_{i}\right)=G_{r b}^{-}\left(\boldsymbol{\theta}_{i}\right)$. Hence, if $G_{r b}\left(\boldsymbol{\theta}_{i}\right) \leqslant 0, N_{f}^{+}, N_{f}^{r b}$ and $N_{f}^{-}$are increased by one altogether.

- If $\mathbf{u}_{r b}\left(\boldsymbol{\theta}_{i}\right)$ is computed from the current reduced basis, $G_{r b}^{-}\left(\boldsymbol{\theta}_{n+1}\right) \leqslant 0, G_{r b}^{+}\left(\boldsymbol{\theta}_{n+1}\right)>0$, and $\varepsilon_{G}\left(\theta_{n+1}\right)<\tau$, then the failure state of the structure is not determined accurately by the reduced basis approximation and only the upper bound $N_{f}^{+}$is increased by one (the value of $N_{f}^{r b}$ being modified accordingly to the value of $G_{r b}\left(\boldsymbol{\theta}_{i}\right)$ ).

The reduced basis failure probability $\hat{P}_{f}^{r b}$ and the bounds $\hat{P}_{f}^{-}$and $\hat{P}_{f}^{+}$of the failure probability $\hat{P}_{f}$ are then easily computed

$$
\hat{P}_{f}^{r b}=\frac{N_{f}^{r b}}{N_{M C}}, \hat{P}_{f}^{+}=\frac{N_{f}^{+}}{N_{M C}}, \hat{P}_{f}^{-}=\frac{N_{f}^{-}}{N_{M C}} .
$$

\section{NUMERICAL EXAMPLES}

\subsection{Example 1}

The first example is a plate with two rectangular holes proposed in [21]. The structure is submitted, in plane strain, to a normal traction $P=20 \mathrm{MPa}$ applied along the vertical edge, and the plate is considered to be composed of three different materials. The symmetry of the problem allows to study only one-fourth of the plate as shown on Figure 1. The Poisson ratios are fixed $v_{1}=v_{2}=v_{3}=0.30$. The Young's moduli $E_{i}$ are random independent variables such that their cumulative distribution function is uniform on [184.5, 225.5] GPa. The QoI considered here is the average displacement on the line $L_{\omega}$.

$$
Q(\mathbf{u}(\boldsymbol{\theta}), \boldsymbol{\theta})=\frac{1}{\operatorname{mes}\left(L_{\omega}\right)} \int_{L_{\omega}} \mathbf{u}(\boldsymbol{\theta}) \cdot \mathbf{n}_{\omega} d l .
$$

The limit state function is given by

$$
G(\boldsymbol{\theta})=\bar{u}-Q(\boldsymbol{\theta})
$$

where $\bar{u}$ is set to $10^{-4}$. Figure 2 shows the mesh used for the FE analysis. A direct Monte Carlo simulation is performed, with the complete FE model, using $n_{M C}=1000$ as a reference value. The failure

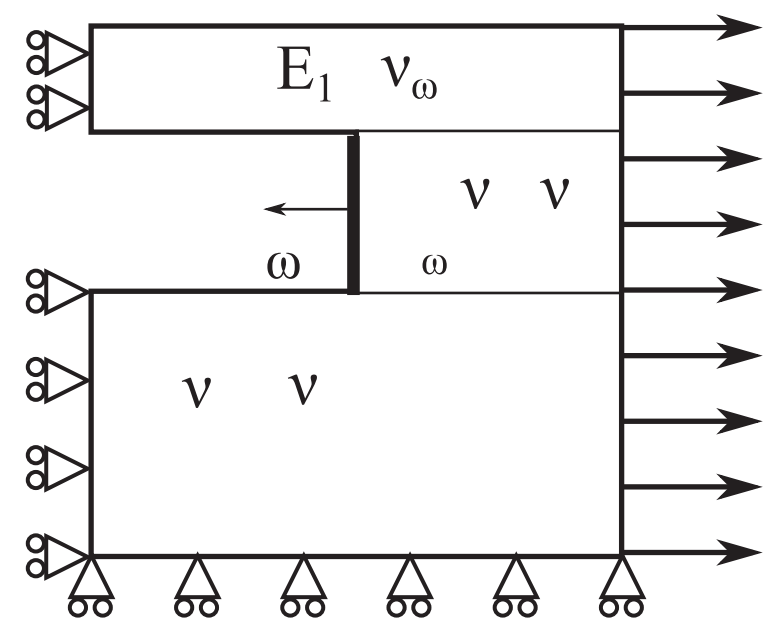

Figure 1. Example 1: thick plate studied. 


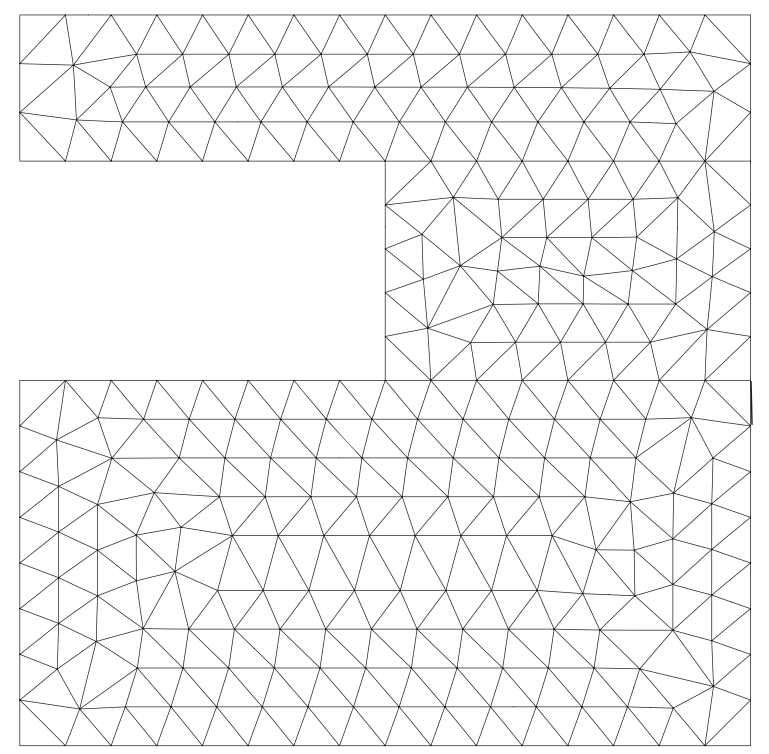

Figure 2. Example 1 mesh: six-node triangular elements - 2122 DoF.

Table I. Example 1: computed failure probability and bounds.

\begin{tabular}{lrrrrrc}
\hline$\tau$ & 0.1 & 0.05 & $10^{-2}$ & $10^{-3}$ & $10^{-4}$ & Reference \\
\hline$\hat{P}_{f}^{r b}\left(10^{-2}\right)$ & 6.7 & 9.1 & 8.5 & 8.5 & 8.5 & 8.5 \\
$\hat{P}_{f}^{+}\left(10^{-2}\right)$ & 24.1 & 10.9 & 8.8 & 8.6 & 8.5 & - \\
$\hat{P}_{f}^{-}\left(10^{-2}\right)$ & 1.6 & 7.5 & 8.4 & 8.5 & 8.5 & - \\
$N_{s}^{\max }$ & \multicolumn{1}{c}{2} & \multicolumn{1}{c}{3} & 3 & 4 & 5 & - \\
\hline
\end{tabular}

probability obtained is $\hat{P}_{f}=8.5 \times 10^{-2}$ and the $95 \%$ confidence interval is $\left[6.77 \times 10^{-2}, 10.2 \times 10^{-2}\right]$. The coefficient of variation $\delta$ obtained from Equation (16) is $\delta=10.8 \%$ and shows that the number of Monte Carlo simulations is sufficient for this example.

Five Monte Carlo simulations $\left(n_{M C}=1000\right)$ are then performed with the reduced basis algorithm for different values of the tolerance $\tau$. For each simulation, we use the same set of random values for the Young's moduli. The results are shown in Table I where the fixed tolerance $\tau$, the failure probability computed with the reduced basis approach $\hat{P}_{f}^{r b}$, its upper and lower bounds $\hat{P}_{f}^{+}$and $\hat{P}_{f}^{-}$, and the maximum number of computed snapshots $N_{s}^{\max }$ have been reported. The number of elements in the basis increases when the tolerance $\tau$ decreases. However, the same number of elements in the basis does not lead to the same bounds as it is shown for $\tau=0.05$ and $\tau=0.01$ where the number of elements in the basis is 3 and the obtained bounds are clearly different. It can be observed that five snapshots are sufficient to obtain a gap between the bounds equal to zero and thus to ensure that the failure probability computed by reduced basis approach is equal to the failure probability that will be obtained by an FE approach. (The elements of the generated reduced basis are shown in Figure 3). That means that the whole Monte Carlo algorithm requires only five complete FE computations. If we take into account that the $95 \%$ confidence interval is $\left[6.77 \times 10^{-2}, 10.2 \times 10^{-2}\right]$, it is clear that obtaining an exact evaluation of $\hat{P}_{f}$ is an overkill and that tolerance $\tau=10^{-2}$ is sufficient to obtain reasonable bounds for $\hat{P}_{f}$ (i.e., $\hat{P}_{f}^{+}-\hat{P}_{f}^{-}$is small compared with the $95 \%$ confidence interval).

The evolution of the number of snapshots as a function of the number of Monte Carlo throws for different values of $\tau$ is shown in Figure 4. 

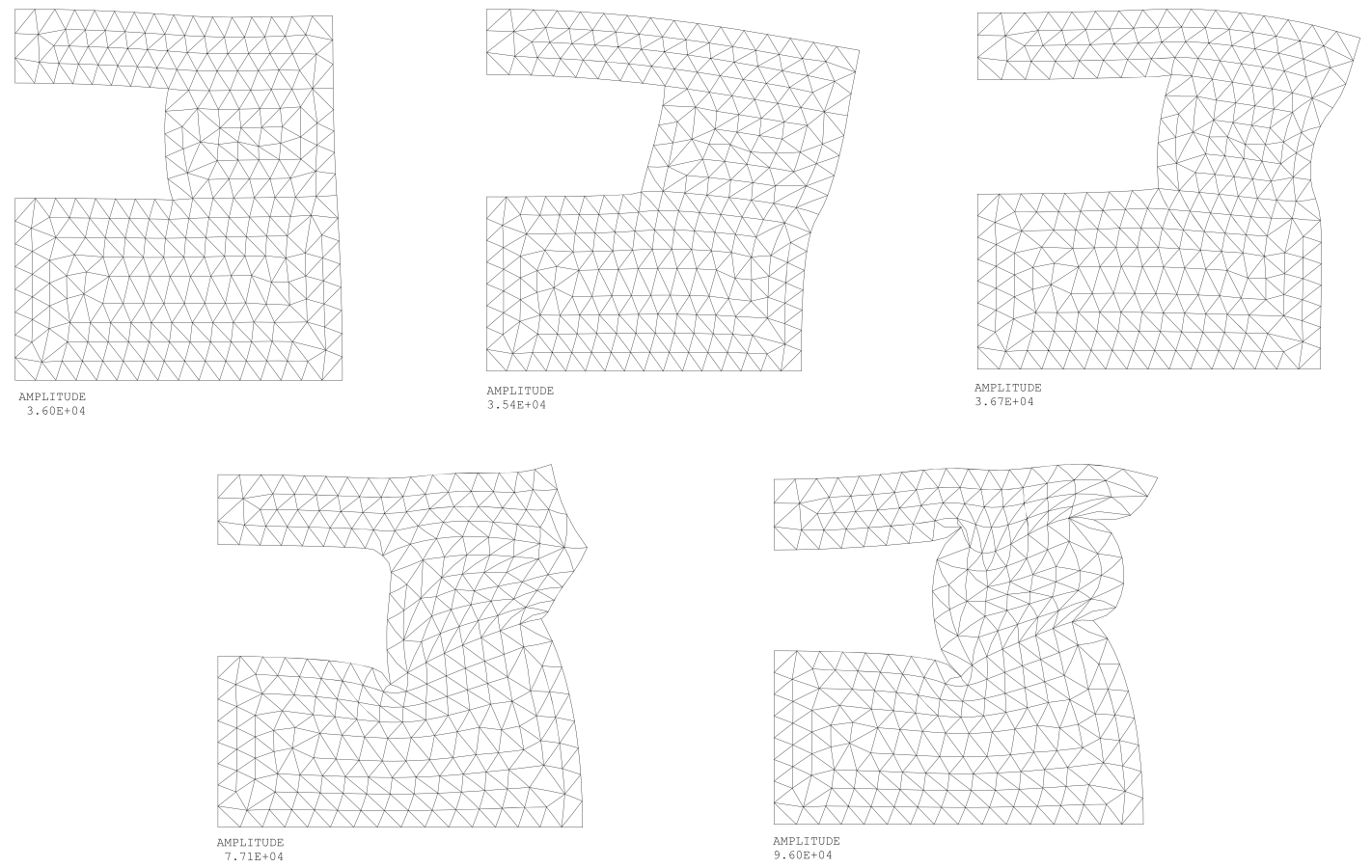

Figure 3. Example 1: reduced basis.

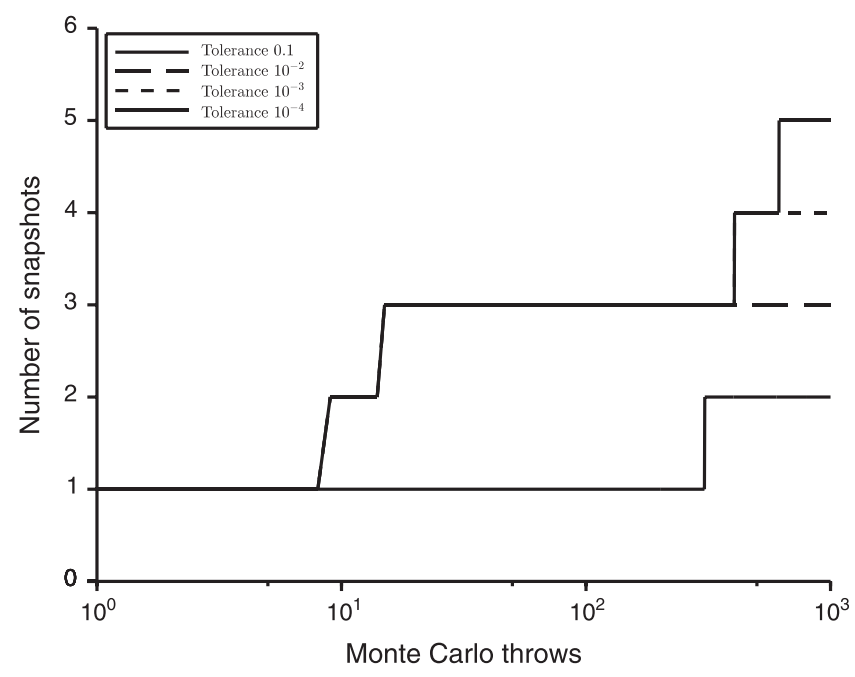

Figure 4. Example 1: number of elements in the reduced basis as a function of the number of Monte Carlo throws.

\subsection{Example 2}

The second example concerns a composite structure composed of two layers linked by an interface (Figure 5). The structure is submitted, in plane strain, to a uniform normal traction $P$ applied along the lower and upper surfaces. We study the probability of propagation of a finite crack of length $a$. The deterministic parameters are the value of the traction $P=1010^{6}$, the resistance to crack propagation $K_{c}=510^{6}$, the crack length $a=0.15$, the length of the structure $L=0.6$, the thickness of the layers $e_{L}=0.14$, the thickness of the interface $e_{I}=0.02$, and the radius of the fibers $r=0.05$ 


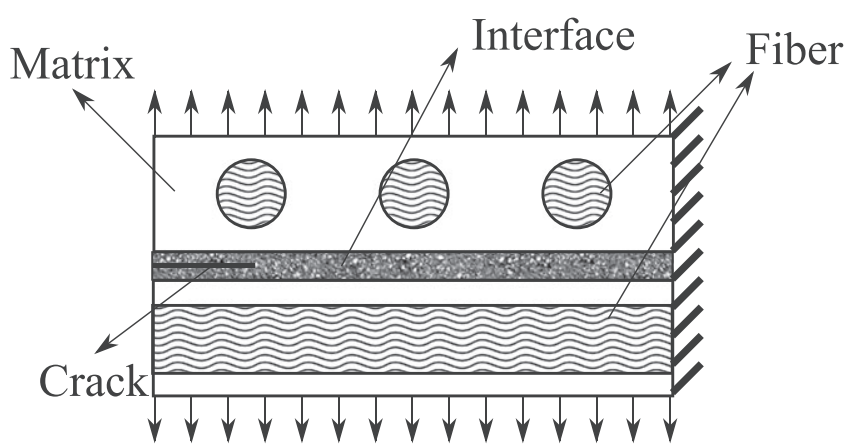

Figure 5. Example 2: composite structure studied.

Table II. Example 2: statistical properties of random variables.

\begin{tabular}{lllll}
\hline Random variable & Distribution & Mean & Minimum & Maximum \\
\hline$E_{F i}$ & Uniform & $1.010^{11}$ & $0.910^{11}$ & $1.110^{11}$ \\
$E_{M}$ & Uniform & $1.010^{10}$ & $0.910^{10}$ & $1.110^{10}$ \\
$E_{I}$ & Uniform & $1.010^{9}$ & $0.910^{9}$ & $1.110^{9}$ \\
\hline
\end{tabular}

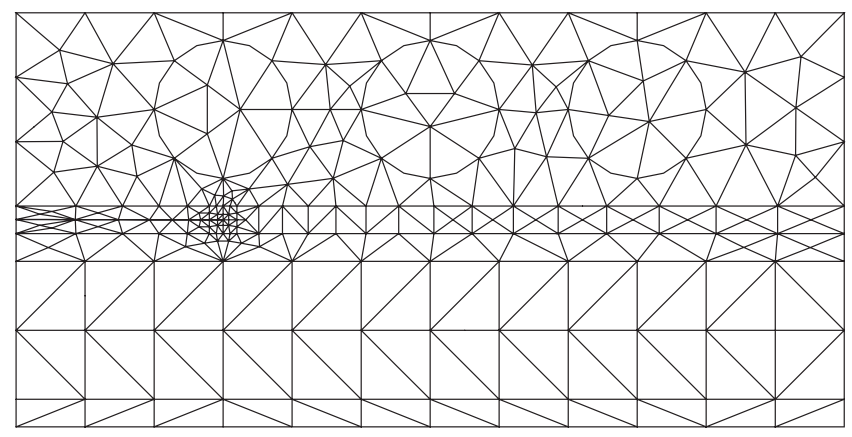

Figure 6. Example 2 mesh: six-node triangular elements - 1800 DoF.

and their positions. The random parameters, as defined in Table II, are the Young's moduli of the materials $\boldsymbol{\theta}=\left(E_{F 1}, E_{F 2}, E_{F 3}, E_{F 4}, E_{M}, E_{I}\right)$ (where $F_{i}$ denotes the fibers, $M$ denotes the matrix, and $I$ denotes the interface). The Poisson ratios are set to 0.3. Figure 6 shows the six-node triangular mesh used for the FE analysis.

Let us denote by $K_{I}(\theta)$ the stress intensity factor at the crack tip. The limit state function is given by

$$
G(\theta)=K_{c}-K_{I}(\theta)
$$

The calculation of the $K_{I}$ consists in an integral around an arbitrary crown $\omega$ defined in the interface around the tip of the crack

$$
K_{I}(\boldsymbol{\theta})=Q(\mathbf{u}(\boldsymbol{\theta}), \boldsymbol{\theta})=\int_{\omega}\left(\mathbf{C}\left(E_{I}\right) \varepsilon\left(\phi \tilde{\mathbf{u}}^{I}\right)-\phi \tilde{\boldsymbol{\sigma}}^{I}\left(E_{I}\right)\right): \varepsilon(\mathbf{u}(\boldsymbol{\theta})) d \omega-\int_{\omega} \tilde{\boldsymbol{\sigma}}^{I}\left(E_{I}\right) \operatorname{grad}(\phi) \cdot \mathbf{u}(\boldsymbol{\theta}) d \omega,
$$

where $\tilde{\boldsymbol{\sigma}}^{I}$ and $\tilde{\mathbf{u}}^{I}$ are singular analytical solutions computed at the crack tip and $\phi$ a function defined on $\omega$ (for further details, see [20,42]). Because of linearity property, it is easy to see that the extractor 
Table III. Example 2: computed failure probability and bounds.

\begin{tabular}{lrlll}
\hline$\tau$ & $10^{-1}$ & $10^{-2}$ & $10^{-3}$ & $10^{-4}$ \\
\hline$\hat{P}_{f}^{r b}\left(10^{-2}\right)$ & 5.90 & 5.65 & 5.65 & 5.65 \\
$\hat{P}_{f}^{+}\left(10^{-2}\right)$ & 36.55 & 6.10 & 5.65 & 5.65 \\
$\hat{P}_{f}^{-}\left(10^{-2}\right)$ & \multicolumn{1}{c}{0.10} & 4.85 & 5.55 & 5.65 \\
$N_{s}^{\max }$ & \multicolumn{1}{c}{3} & 7 & 9 & 11 \\
\hline
\end{tabular}

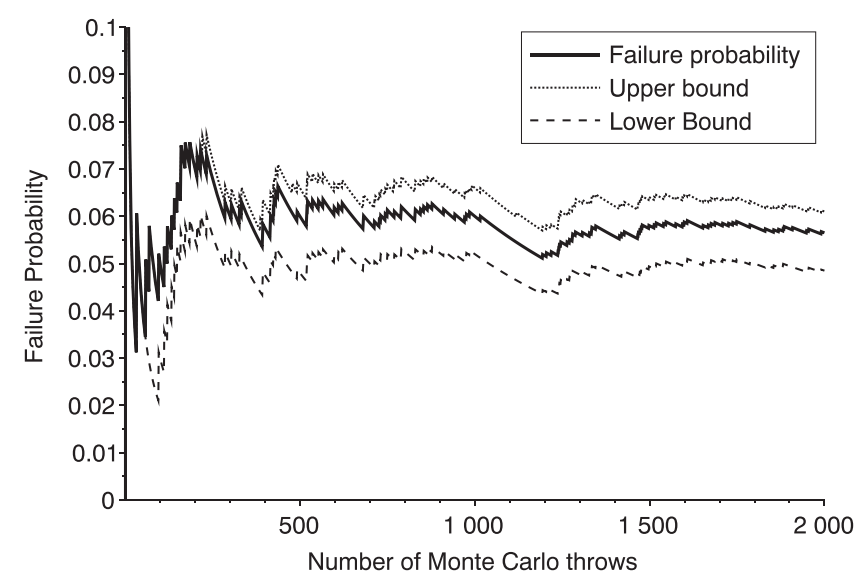

Figure 7. Example 2: failure probability $\hat{P}_{f}^{r b}, \hat{P}_{f}^{+}$, and $\hat{P}_{f}^{-}$as a function of Monte Carlo throws for $\tau=10^{-2}$.

that defines the output of interest can be decomposed as the sum of functions of $\boldsymbol{\theta}$ multiplied by deterministic functions of $\mathbf{x}$.

$$
Q(\mathbf{u}(\boldsymbol{\theta}), \boldsymbol{\theta})=\left(\frac{E_{I}}{E_{\text {ref }}}\right)\left(\int_{\omega} \bar{\Sigma}_{\Sigma}: \varepsilon(\mathbf{u}(\boldsymbol{\theta})) d \omega-\int_{\omega} \overline{\mathbf{b}}_{\Sigma} \cdot \mathbf{u}(\boldsymbol{\theta}) d \omega\right),
$$

where $E_{r e f}>0$ is an arbitrary scalar and

$$
\bar{\Sigma}_{\Sigma}=\left(\mathbf{C}\left(E_{r e f}\right) \varepsilon\left(\phi \tilde{\mathbf{u}}^{I}\right)-\phi \tilde{\boldsymbol{\sigma}}^{I}\left(E_{r e f}\right)\right) \text { and } \overline{\mathbf{b}}_{\Sigma}=\tilde{\boldsymbol{\sigma}}^{I}\left(E_{r e f}\right) \operatorname{grad}(\phi) .
$$

A direct Monte Carlo simulation is performed, with the complete FE model, using $n_{M C}=2000$ as a reference value. The failure probability obtained is $\hat{P}_{f}=5.65 \times 10^{-2}$ and the $95 \%$ confidence interval is $\left[4.64 \times 10^{-2}, 6.66 \times 10^{-2}\right]$. Then, four Monte Carlo simulations $\left(n_{M C}=2000\right)$ are performed with the reduced basis algorithm for different values of the tolerance $\tau$. For each simulation, we use the same set of random values for the Young's moduli. The results are shown in Table III where the fixed tolerance $\tau$, the failure probability computed with the reduced basis approach $\hat{P}_{f}^{r b}$, its upper and lower bounds $\hat{P}_{f}^{+}$and $\hat{P}_{f}^{-}$, and the maximum number of computed snapshots $N_{s}^{\max }$ have been reported.

Figure 7 represents the evolution of the failure probability $\hat{P}_{f}^{r b}$, its upper bound $\hat{P}_{f}^{+}$, and its lower bound $\hat{P}_{f}^{-}$as a function of the Monte Carlo throws for a tolerance $\tau=10^{-2}$. This tolerance is sufficient to obtain bounds smaller than the bounds obtained for the $95 \%$ confidence interval.

Table IV shows the evolution of the failure probability and of the number of the elements in the reduced basis when the interval of the uniform probability distribution $E_{\max }-E_{\min }$ increases. The mean value proposed for each material in Table II is conserved, and we introduce a parameter $\lambda$ such that $E_{\max }=(1+\lambda) E_{\text {mean }}$ and $E_{\min }=(1-\lambda) E_{\text {mean }}$. Failure probability predictability increases with $\lambda$ as well as the number of elements in the reduced basis. As shown in Equation (16), for a given 
Table IV. Example 2: computed failure probability and bounds.

\begin{tabular}{lccccc}
\hline$\lambda$ & 0.1 & 0.2 & 0.4 & 0.6 & 0.8 \\
\hline$\hat{P}_{f}^{r b}\left(10^{-2}\right)$ & 5.65 & 21.9 & 36.5 & 43.0 & 47.7 \\
$\hat{P}_{f}^{+}\left(10^{-2}\right)$ & 6.10 & 22.6 & 37.6 & 43.8 & 48.4 \\
$\hat{P}_{f}^{-}\left(10^{-2}\right)$ & 4.85 & 21.2 & 35.3 & 42.0 & 46.6 \\
Upper bound of the confidence interval $\left(10^{-2}\right)$ & 6.66 & 23.7 & 38.6 & 45.1 & 45.5 \\
Lower bound of the confidence interval $\left(10^{-2}\right)$ & 4.64 & 20.0 & 34.3 & 40.8 & 50.6 \\
$N_{s}^{\max }$ & 7 & 8 & 11 & 14 & 20 \\
\hline
\end{tabular}

Table V. Example 2: computed failure probability and bounds.

\begin{tabular}{lcccc}
\hline$K_{I C}\left(10^{6}\right)$ & 4.90 & 5.00 & 5.05 & \multicolumn{2}{c}{5.10} \\
\hline$n_{M C}$ & 2000 & 2000 & 5000 & 80,000 \\
$\hat{P}_{f}^{r b}\left(10^{-2}\right)$ & 24.8 & 5.65 & 1.56 & 0.103 \\
$\hat{P}_{f}^{+}\left(10^{-2}\right)$ & 29.7 & 6.10 & 2.14 & 0.224 \\
$\hat{P}_{f}^{-}\left(10^{-2}\right)$ & 21.8 & 4.85 & 1.16 & 0.028 \\
Upper bound of the confidence interval $\left(10^{-2}\right)$ & 26.6 & 6.66 & 1.90 & 0.125 \\
Lower bound of the confidence interval $\left(10^{-2}\right)$ & 22.8 & 4.64 & 1.22 & 0.080 \\
$N_{s}^{\max }$ & 7 & 6 & 6 & 6 \\
\hline
\end{tabular}

coefficient of variation $\delta$, the required number of Monte Carlo throws increases with the reduction of the failure probability. Table $\mathrm{V}$ shows the evolution of the failure probability and the evolution of the number of the elements in the reduced basis when the resistance to crack propagation increases, as well as the number of Monte Carlo throws used to take into account the reduction of the failure probability (for this simulation, we use the statistical data given in Table II and $\tau$ is set to $10^{-2}$ ). It can be seen that there is no increase in the number of elements in the reduced basis and that six FE computations are sufficient to accurately estimate the failure probability.

\section{CONCLUSIONS}

This paper describes a new strategy to generate a reduced basis in the context of a reliability analysis based on the Monte Carlo algorithm. The reduced basis vectors are chosen in order to control the quality of the estimation of the limit state function. An error estimator on the QoI based on the error in the constitutive relation is used to compute an upper bound as well as a lower bound of the failure probability obtained by the Monte Carlo algorithm. The presented examples in 2D elasticity show that few FE computations are necessary to generate a reduced basis that leads to bounds of the same order of magnitude as a $95 \%$ confidence interval.

\section{ACKNOWLEDGEMENTS}

This study was supported by the Publishing Arts Research Council (981846389).

\section{REFERENCES}

1. Ditlevsen O, Madsen HO. Structural Reliability Methods. Wiley: New York, 1996.

2. Lemaire M. Fiabilité des Structures. Hermes: Paris, 2005.

3. Der Kiureghian A, de Stefano M. Efficient algorithm for second-order reliability analysis. Journal of Engineering Mechanics 1991; 12(117):37-49. 
4. Gallimard L. Error bounds for the reliability index in finite element reliability analysis. International Journal for Numerical Methods in Engineering 2011; 87(8):781-794.

5. Gallimard L, Vidal P, Polit O. Coupling finite element and reliability analysis through proper generalized decomposition model reduction. International Journal for Numerical Methods in Engineering 2013; 95(13):1079-1093.

6. Hasofer AM, Lind NC. An exact and invariant first order reliability format. Journal of Engineering Mechanics, ASCE 1974; 100(1):111-121.

7. Haukaas T, Der Kiureghian A. Strategies for finding the design point in non-linear finite element reliability analysis. Probabilistic Engineering Mechanics 2006; 21(2):133-147.

8. Rackwitz R, Fiessler B. Structural reliability under random load sequences. Computer and Structures 1979; 5(9):484-494.

9. Zhang Y, Der Kiureghian A. Two improved algorithms for reliability analysis. In Proceeding of 6th IFIP WG7.5 Reliability and Optimization of Structural, Assisi, Italy; 297-304, 1994.

10. Berveiller M, Sudret B, Lemaire M. Stochastic finite elements: a non intrusive approach by regression. European Journal of Mechanics 2006; 15(1-3):81-92.

11. Blatman G, Sudret B. An adaptive algorithm to build up sparse polynomial chaos expansions for stochastic finite element analysis. Probabilistic Engineering Mechanics 2010; 25(2):183-197.

12. Colombi P, Faravelli L. Stochastic finite elements via response surface: fatigue crack growth problems. In Probabilistic Methods for Structural Design, Springer, Netherlands, Dordrecht; 313-338, 1997.

13. Pendola M, Mohamed A, Lemaire M, Hornet P. Combination of finite element and reliability methods in nonlinear fracture mechanics. Reliability Engineering \& System Safety 2000; 70(1):15-27.

14. Sudret Bruno, Der Kiureghian A. Comparison of finite element reliability methods. Probabilistic Engineering Mechanics 2002; 17:337-348.

15. Boyaval S, Le Bris C, Lelièvre T, Maday Y, Nguyen NC, Patera AT. Reduced basis techniques for stochastic problems. Archives of Computational Methods in Engineering 2010; 17:435-454.

16. Boyaval S, Le Bris C, Maday Y, Nguyen NC, Patera AT. Reduced basis approach for variational problems with stochastic parameters: application to heat conduction with variable Robin coefficient. Computer Methods in Applied Mechanics and Engineering 2009; 198(41-44):3187-3206.

17. Florentin E, Dìez P. Adaptive reduced basis strategy based on goal oriented error assessment for stochastic problems. Computer Methods in Applied Mechanics and Engineering 2012; 225-228:116-127.

18. Grigoriu M. Reduced order models for random functions application to stochastic problems. Applied Mathematical Modelling 2009; 33:161-175.

19. Mohan PS, Nair PB, Keane AJ. Multi-element stochastic reduced basis methods. Computer Methods in Applied Mechanics and Engineering 2008; 197:1495-1506.

20. Gallimard L, Panetier J. Error estimation of stress intensity factors for mixed-mode cracks. International Journal for Numerical Methods in Engineering 2006; 68(3):299-316.

21. Peraire J, Patera A, Ladevèze P, Oden JT. Bounds for linear-functional outputs of coercive partial differential equations: local indicators and adaptive refinement. In Advances in Adaptive Computational Methods, Elsevier; 199-216, 1998.

22. Prudhomme S, Oden JT. On goal-oriented error estimation for elliptic problems: application to the control of pointwise errors. Computer Methods in Applied Mechanics and Engineering 1999; 176:313-331.

23. Almeida RC, Oden JT. Solution verification, goal-oriented adaptive methods for stochastic advection-diffusion problems. Computer Methods in Applied Mechanics and Engineering 2010; 199:2472-2486.

24. Butler T, Dawson C, Widley T. A posteriori error analysis of stochastic differential equation using polynomial chaos expansion. SIAM Journal on Scientific Computing 2011; 33:1267-1291.

25. Chamoin L, Florentin E, Pavot S, Visseq V. Robust goal-oriented error estimation based on the constitutive relation error for stochastic problems. Computers and Structures 2012; 106107(0):189-195.

26. Gallimard L, Ryckelynck D. A posteriori global error estimator based on the error in the constitutive relation for reduced basis approximation of parametrized linear elastic problems. Applied Mathematical Modelling 2016; 40:4271-4284.

27. Kerfriden P, Rodenas JJ, Bordas SPA. Certification of projection-based reduced order modelling in computational homogenisation by the constitutive relation error. International Journal for Numerical Methods in Engineering 2014; 97(6):395-422.

28. Ladevèze $\mathrm{P}$, Chamoin L. On the verification of model reduction methods based on the proper generalized decomposition. Computer Methods in Applied Mechanics and Engineering 2011; 200:2032-2047.

29. Moitinho de Almeida JP. A basis for bounding the errors of proper generalized decomposition in solids mechanics. International Journal for Numerical Methods in Engineering 2013; 94:961-984.

30. Rozza G, Huynh DBP, Patera AT. Reduced basis approximation and a posteriori error estimation for affinely parametrized elliptic coercive partial differential equations. Archives of Computational Methods in Engineering 2008; 15:229-275.

31. Ghanem RG, Spanos PD. Stochastic Finite Elements: A Spectral Approach, Revised Edition. Dover: New York, 2003.

32. Mohamed A, Lemaire M, Mitteau J-C, Meister E. Finite element and reliability: a method for compound variables - application on a cracked heating system. Nuclear Engineering and Design 1998; 185(2):185-202.

33. Ladevèze $\mathrm{P}$, Rougeot $\mathrm{P}$, Blanchard $\mathrm{P}$, Moreau JP. Local error estimators for finite element analysis. Computer Methods in Applied Mechanics and Engineering 1999; 176:231-246. 
34. Ohnimus S, Stein E, Walhorn E. Local error estimates of FEM for displacements and stresses in linear elasticity by solving local Neumann problems. International Journal for Numerical Methods in Engineering 2001; 52:727-746.

35. Prudhomme S, Oden JT, Westermann T, Bass J, Botkin ME. Practical methods for a posteriori error estimation in engineering applications. International Journal for Numerical Methods in Engineering 2003; 56:1193-1224.

36. Hoang KC, Kerfriden P, Bordas SPA. A fast, certified and tuning free two-field reduced basis method for the metamodelling of affinely-parametrised elasticity problems. Computer Methods in Applied Mechanics and Engineering 2016; 298: $121-158$.

37. Machiels L, Maday Y, Patera AT. Output bounds for reduced-order approximations of elliptic partial differential equations. Computer Methods in Applied Mechanics and Engineering 2001; 190:3413-3426.

38. Ryckelynck David, Gallimard Laurent, Jules Samuel. Estimation of the validity domain of hyper-reduction approximations in generalized standard elastoviscoplasticity. Advanced Modeling and Simulation in Engineering Sciences 2015; 2(1):1-19.

39. Ladevèze P, Leguillon D. Error estimate procedure in the finite element method and application. SIAM Journal on Numerical Analysis 1983; 20(3):485-509.

40. Gallimard L. A constitutive relation error estimator based on traction-free recovery of the equilibrated stress. International Journal for Numerical Methods in Engineering 2009; 78(4):460-482.

41. Gallimard L. Evaluation of the local quality of the von Mises's stress and L2-norm of the stress. Engineering Computations 2006; 68(7/8):876-897.

42. Gallimard L. Error control in form reliability analysis. European Journal of Computational Mechanics 2012; 21(3-6):231-241. 